JOURNAL OF OPTIMIZATION, DIFFERENTIAL EQUATIONS AND THEIR APPLICATIONS (JODEA) Volume 26, Issue 2, December 2018, pp. 13-36, DOI $10.15421 / 141808$

ISSN (print) 2617-0108

ISSN (on-line) $\mathrm{xxxx}-\mathrm{xxxx}$

\title{
ON INDIRECT APPROACH TO THE SOLVABILITY OF QUASI-LINEAR DIRICHLET ELLIPTIC BOUNDARY VALUE PROBLEM WITH BMO-ANISOTROPIC P-LAPLACIAN
}

\author{
Peter I. Kogut, Olha P. Kupenko ${ }^{\dagger \ddagger}$
}

\begin{abstract}
We study here Dirichlet boundary value problem for a quasi-linear elliptic equation with anisotropic $p$-Laplace operator in its principle part and $L^{1}$-control in coefficient of the low-order term. As characteristic feature of such problem is a specification of the matrix of anisotropy $A=A^{\text {sym }}+A^{\text {skew }}$ in $B M O$-space. Since we cannot expect to have a solution of the state equation in the classical Sobolev space $W_{0}^{1, p}(\Omega)$, we specify a suitable functional class in which we look for solutions and prove existence of weak solutions in the sense of Minty using a non standard approximation procedure and compactness arguments in variable spaces.
\end{abstract}

Key words: Anisotropic $p$-Laplacian, approximation procedure, weak solutions, BMOcoefficients.

2010 Mathematics Subject Classification: 35J92, 30H35, 35D30.

Communicated by Prof. O. V. Kapustyan

\section{Introduction}

In this paper we deal with the following boundary value problem

$$
\left\{\begin{array}{c}
-\Delta_{p}(A, y)+|y|^{p-2} y u=-\operatorname{div} f \quad \text { in } \Omega, \quad y=0 \quad \text { on } \partial \Omega, \\
u \in L^{1}(\Omega), \quad u(x) \geq 0 \text { a.e. in } \Omega,
\end{array}\right.
$$

where

$$
-\Delta_{p}(A, y)=-\operatorname{div}\left(|(\nabla y, A \nabla y)|^{\frac{p-2}{2}} A \nabla y\right)
$$

is the anisotropic $p$-Laplacian, $2 \leq p<+\infty, A$ is the matrix of anisotropy, $y_{d} \in L^{2}(\Omega)$ and $f \in L^{\infty}\left(\Omega ; \mathbb{R}^{N}\right)$ are given distributions.

The interest to elliptic equations whose principal part is an anisotropic $p$ Laplace operator arises from various applied contexts related to composite materials such as nonlinear dielectric composites, whose nonlinear behavior is modeled by the so-called power-low (see, for instance, $[1,21]$ and references therein). From mathematical point of view, the interest of anisotropic $p$-Laplacian lies on its

\footnotetext{
*Department of Differential Equations, Oles Honchar Dnipro National University, 72, Gagarin av., Dnipro, 49010, Ukraine, p.kogut@i.ua

${ }^{\dagger}$ Department of System Analysis and Control, National Mining University, 19, Yavornitskii av., 49005 Dnipro

${ }^{\ddagger}$ Institute of Applied System Analysis, National Academy of Sciences and Ministry of Education and Science of Ukraine, 37/35, Peremogy av., IASA, 03056 Kyiv, Ukraine, kupenko.olga@gmail.com

(C) P. I. Kogut, O.P. Kupenko, 2018.
} 
nonlinearity and an effect of degeneracy, which turns out to be the major difference from the standard Laplacian on $\mathbb{R}^{N}$. As characteristic feature of boundary value problem (1.1) is a specification of the matrix of anisotropy $A=B+D$, where $B:=A^{\text {sym }}=\left(A+A^{t}\right) / 2$ and $D:=A^{\text {skew }}=\left(A-A^{t}\right) / 2$, and the control $u \in L^{1}(\Omega)$. In particular, we assume that the matrix $A$ is such that

$$
\alpha^{2}(x) I \leq B(x) \leq \beta^{2}(x) I \quad \text { a. e. in } \Omega,
$$

where $\alpha, \beta \in L^{1}(\Omega), \beta(x) \geq \alpha(x) \geq 0$ almost everywhere in $\Omega, \alpha \notin L^{\infty}(\Omega)$, $\alpha^{-1} \in L^{1}(\Omega)$, and $\alpha, \alpha^{-1}$, and $\beta$ extended by zero outside of $\Omega$ are in $B M O\left(\mathbb{R}^{N}\right)$.

We note that these assumptions on the class of admissible matrices are essentially weaker than they usually are in the literature (see, for instance, [8, 9, $11,19,20])$. In fact, we deal with the Dirichlet boundary value problem (BVP) for degenerate anisotropic elliptic equation with unbounded coefficients in its principal part and with $L^{1}$-bounded control in the coefficient of the low-order term. It is well-known that such BVP can exhibit the so-called Lavrentieff phenomenon, non-uniqueness of the weak solutions as well as other surprising consequences (see, for instance, $[2,4]$ ). As a result, the existence, uniqueness, and variational properties of the weak solution to the above BVP usually are drastically different from the corresponding properties of solutions to the elliptic equations with coercive $L^{\infty}$-matrices of anisotropy (we refer to $[6,26-28,31]$ for the details and other results in this field). Another distinguishing feature of the boundary value problem (3.1)-(3.2) is the fact that the skew-symmetric part $D$ of the matrix $A$ is merely measurable and its sub-multiplicative norm belongs to the $B M O$-space (rather than the space $L^{\infty}(\Omega)$ ). This circumstance can entail a number of pathologies with respect to the standard properties of BVPs for elliptic equations with anisotropic $p$-Laplacian even with 'a good' symmetric part $A$ and a smooth right-hand side $f$. In particular, the unboundedness of the skew-symmetric part of matrix $A \in \mathfrak{M}_{a d}$ can have a reflection in non-uniqueness of weak solutions to the corresponding boundary value problem. For more details and other types of solutions to elliptic equations with unbounded coefficients we refer to $[7,14-16,33]$. So, in contrast to the paper [32], where the author consider the case of well-posed Dirichlet boundary value problem for a quasi-linear elliptic equation with unbounded coefficients in its principal part, we deal with an ill-posed boundary value problem.

We introduce a special functional space $\mathbb{X}_{u, B}$ related to a given control $u$ and symmetric part $B$ of matrix $A$, and prove (see Theorem 4.1) that the original boundary value problem admits weak solutions in the sense of Minty. Moreover, we show that for every control $u \in L^{1}(\Omega)$, a weak solutions (in the sense of Minty) to the corresponding BVP can be obtained as the limit of solutions to coercive problems with bounded coefficients, using any $L^{\infty}$-approximation of $B M O$-matrix $A$. Such solutions are called approximation solutions in [33]. Their characteristic feature is the fact that they lay in variable space $\mathbb{X}_{u, B}$ and, in general, do not satisfy the energy equality but rather some energy inequality. We also derive a priori estimates for such solutions that do not depend on the skew-symmetric part $D$ of matrix $A$. As a bi-product of our approach, we derive the conditions 
guaranteeing the equality $H_{0, B}^{1, p}(\Omega)=W_{0, B}^{1, p}(\Omega)$, i.e. we establish the density of smooth compactly supported functions in $W_{0, B}^{1, p}(\Omega)$.

\section{Notation and Preliminaries}

Let $\Omega$ be a bounded open subset of $\mathbb{R}^{N}(N \geq 1)$ with a Lipschitz boundary. Let $p$ be a real number such that $2 \leq p<\infty$, and let $q=p /(p-1)$ be the conjugate of $p$. Let $\mathbb{M}^{N}$ be the set of all $N \times N$ real matrices. We denote by $\mathbb{S}_{\text {skew }}^{N}$ and $\mathbb{S}_{s y m}^{N}$ the set of all skew-symmetric and symmetric matrices, respectively. We always identify each matrix $A \in \mathbb{M}^{N}$ with the decomposition $A=B+D$, where $B:=\frac{1}{2}\left(A+A^{t}\right) \in \mathbb{S}_{\text {sym }}^{N}$ and $D:=\frac{1}{2}\left(A-A^{t}\right) \in \mathbb{S}_{\text {skew }}^{N}$. Moreover, applying the Cholesky method to the symmetric part of matrix $A$ (see Isaacson and Keller [30]), we deduce the existence of a lower triangular matrix $L$ such that $B(x):=$ $\frac{1}{2}\left(A(x)+A^{t}(x)\right)=L^{t}(x) L(x)$. In what follows, by matrix norm in $\mathbb{M}^{N}$ we mean a sub-multiplicative norm

$$
\|A\|:=\sup _{\substack{|\xi| \neq 0 \\ \xi \in \mathbb{R}^{N}}}\left\{\frac{|A \xi|}{|\xi|}\right\}=\left(\text { maximal eigenvalue of } A^{t} A\right)^{1 / 2} \text { a.e. in } \Omega .
$$

BMO-Functions Defined on Bounded Domains. We recall that a function $g$ on $\mathbb{R}^{N}$ belongs to the space $B M O\left(\mathbb{R}^{N}\right)$ if $g \in L_{\text {loc }}^{1}\left(\mathbb{R}^{N}\right)$ and

$$
\|g\|_{B M O\left(\mathbb{R}^{N}\right)}:=\sup \frac{1}{|Q|} \int_{Q}\left|g-g_{Q}\right| d x<+\infty
$$

where $g_{Q}=f_{Q} g d x:=\frac{1}{|Q|} \int_{Q} g d x, Q=Q(x, r)$ is a ball centered at $x$ and of radius $\ell(Q)=r$, and the supremum is taken over all balls $Q \subset \mathbb{R}^{N}$. Obviously, $L^{\infty}\left(\mathbb{R}^{N}\right) \subset B M O\left(\mathbb{R}^{N}\right)$. As an example of unbounded function in $B M O\left(\mathbb{R}^{N}\right)$, one can take $\ln |x|$.

For our further analysis, we make use of the following result: if $g \in B M O\left(\mathbb{R}^{N}\right)$ then the John-Nirenberg estimate

$$
f_{Q}\left|g-g_{Q}\right|^{p} d x \leq C_{p, \Omega}\|g\|_{B M O\left(\mathbb{R}^{N}\right)} \quad \text { for all } p \geq 1
$$

holds for any ball $Q \subset \mathbb{R}^{N}$ (see [13]).

Let $L^{1}(\Omega)^{\frac{N(N+1)}{2}}=L^{1}\left(\Omega ; \mathbb{S}_{s y m}^{N}\right)$ be the space of measurable absolutely integrable functions whose values are symmetric matrices. By $B M O\left(\Omega ; \mathbb{S}_{\text {skew }}^{N}\right)$ we denote the space of all skew-symmetric matrices $D=\left[d_{i j}\right]$ (the-so-called matrices of bounded mean oscillation) such that $D \in L^{1}\left(\Omega ; \mathbb{S}_{\text {skew }}^{N}\right)$ and their sub-multiplicative norm extended by zero to the entire $\mathbb{R}^{N}$ is in $B M O\left(\mathbb{R}^{N}\right)$. The similar specification holds for the space $B M O\left(\Omega ; \mathbb{M}^{N}\right)$. 
Matrices with Degenerate Eigenvalues. Let $\alpha, \beta$ be given elements of $L^{1}(\Omega)$ satisfying the conditions

$$
\begin{aligned}
& \alpha^{-1} \in L^{1}(\Omega), \quad \alpha^{-1} \notin L^{\infty}(\Omega), \quad 0 \leq \alpha(x) \leq \beta(x) \text { a.e. in } \Omega, \\
& \alpha, \alpha^{-1}, \beta \text { extended by zero outside of } \Omega \text { are in } B M O\left(\mathbb{R}^{N}\right) .
\end{aligned}
$$

Remark 2.1. As immediately follows from the John-Nirenberg estimate (2.1) and assumption (2.3), we have

$$
\begin{aligned}
\left\|\alpha^{-1}\right\|_{L^{r}(\Omega)}^{r} & \leq 2^{r-1} \int_{\Omega}\left|\alpha^{-1}-\alpha_{Q}^{-1}\right|^{r} d x+2^{r-1}\left(\frac{1}{|Q|} \int_{Q} \alpha^{-1} d x\right)^{r}|\Omega| \\
& \leq 2^{r-1}|Q|\left[f_{Q}\left|\alpha^{-1}-\alpha_{Q}^{-1}\right|^{r} d x+\frac{|\Omega|}{|Q|^{r+1}}\left(\int_{\Omega} \alpha^{-1} d x\right)^{r}\right] \\
& \text { by } \stackrel{(2.1)}{\leq} C_{Q, r}\left(\left\|\alpha^{-1}\right\|_{B M O\left(\mathbb{R}^{N}\right)}+\left\|\alpha^{-1}\right\|_{L^{1}(\Omega)}^{r}\right) \quad \forall r>1 .
\end{aligned}
$$

Here, $Q$ is a ball such that $\Omega \subset Q$, and $\alpha_{Q}^{-1}=f_{Q} \alpha^{-1} d x$. The similar estimates hold true for $\alpha$ and $\beta$. So, we can suppose that $\alpha, \alpha^{-1}, \beta \in L^{r}(\Omega)$ for all $r \geq 1$ provided the conditions (2.2)-(2.3) hold true.

We define the class of matrices $\mathfrak{M}_{a d}$ as follows

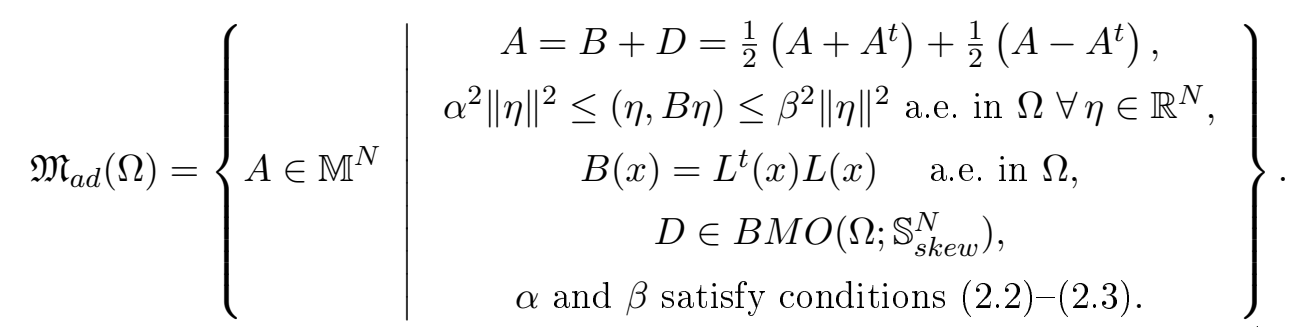

Remark 2.2. Here, in view of the estimate $(\eta, B \eta) \geq \alpha^{2}\|\eta\|^{2}$ a.e. in $\Omega \forall \eta \in \mathbb{R}^{N}$, $L$ is a triangular matrix with positive (a.e. in $\Omega$ ) diagonal elements. Moreover, for a fixed $A \in \mathfrak{M}_{a d}$, conditions (2.2)-(2.5) imply the following inequalities:

$$
\begin{gathered}
\|L\|_{B M O\left(\Omega ; \mathbb{M}^{N}\right)} \leq\|\beta\|_{B M O\left(\mathbb{R}^{N}\right)}<+\infty \\
(B(x) \xi, \xi)=|L(x) \xi|^{2} \leq \beta^{2}(x)|\xi|^{2} \quad \text { a. e. in } \Omega, \forall \xi \in \mathbb{R}^{N} \\
\left|L^{-1}(x) \xi\right|^{2} \leq \alpha^{-2}(x)|\xi|^{2} \quad \text { a. e. in } \Omega, \forall \xi \in \mathbb{R}^{N},
\end{gathered}
$$

and, therefore,

$$
\begin{gathered}
\|L(x)\| \leq \beta(x) \quad \text { and }\left\|L^{-1}(x)\right\| \leq \alpha^{-1}(x) \quad \text { a. e. in } \Omega \\
L, L^{-1} \in B M O\left(\Omega ; \mathbb{M}^{N}\right) .
\end{gathered}
$$


Weighted Sobolev Spaces. To each matrix $A \in \mathfrak{M}_{a d}(\Omega)$ we can formally associate two weighted Sobolev spaces: $W_{0, B}^{1, p}(\Omega)$ and $H_{0, B}^{1, p}(\Omega)$, where $W_{0, B}^{1, p}(\Omega)$ is the set of functions $y \in W_{0}^{1,1}(\Omega)$ for which the norm

$$
\|y\|_{W_{0, B}^{1, p}(\Omega)}=\left(\int_{\Omega}\left(|y|^{p}+|(\nabla y, B \nabla y)|^{\frac{p}{2}}\right) d x\right)^{1 / p}
$$

is finite, and $H_{0, B}^{1, p}(\Omega)$ is the closure of $C_{0}^{\infty}(\Omega)$ with respect to the norm (2.11).

As follows from the definition of the class $\mathfrak{M}_{a d}$ and estimates

$$
\begin{aligned}
\int_{\Omega}|y| d x & \leq\left(\int_{\Omega}|y|^{p} d x\right)^{1 / p}|\Omega|^{1 / q} \leq C\|y\|_{W_{0, B}^{1, p}(\Omega)}, \forall y \in W_{0, B}^{1, p}(\Omega) \\
\int_{\Omega}|\nabla y| d x & \leq\left(\int_{\Omega}|\nabla y|^{p} \alpha^{p} d x\right)^{1 / p}\left(\int_{\Omega} \alpha^{-q} d x\right)^{1 / q} \\
& \leq\left(\int_{\Omega}|(\nabla y, B(x) \nabla y)|^{p / 2} d x\right)^{1 / p}\left\|\alpha^{-1}\right\|_{L^{q}(\Omega)} \leq C\|y\|_{W_{0, B}^{1, p}(\Omega)}
\end{aligned}
$$

the space $W_{0, B}^{1, p}(\Omega)$ is complete with respect to the norm $\|\cdot\|_{W_{0, B}^{1, p}(\Omega)}$. It is clear that $H_{0, B}^{1, p}(\Omega)$ and $W_{0, B}^{1, p}(\Omega)$, for $p \geq 2$, are uniformly convex reflexive Banach spaces such that $H_{0, B}^{1, p}(\Omega) \subseteq W_{0, B}^{1, p}(\Omega)$ (see, for instance [10]). In general, the identity $W_{0, B}^{1, p}(\Omega)=H_{0, B}^{1, p}(\Omega)$ is not always valid (for the corresponding examples, we refer to $[5])$.

Further we make use of the following observation. If we introduce the parameter $p_{s}$ by $p_{s}:=p s /(s+1)<p$ with a certain $s>0$ and use the Hölder inequality with the parameter $r=\frac{s+1}{s}=\frac{p}{p_{s}}>1$, we obtain

$$
\begin{aligned}
\int_{\Omega}|\nabla y|^{p_{s}} d x & =\int_{\Omega}|\nabla y|^{p_{s}} \alpha^{p_{s}} \alpha^{-p_{s}} d x \\
& \leq\left(\int_{\Omega}|\nabla y|^{p} \alpha^{p} d x\right)^{p_{s} / p}\left(\int_{\Omega} \alpha^{-s-1} d x\right)^{1 /(s+1)} \\
& \leq\left(\int_{\Omega}|(\nabla y, B(x) \nabla y)|^{p / 2} d x\right)^{s /(s+1)}\left\|\alpha^{-1}\right\|_{L^{s+1}(\Omega)} \\
& \text { by } \stackrel{(2.4)}{\leq} C\|y\|_{H_{0, B}^{1, p}(\Omega)}^{\frac{p_{s}}{p}}, \\
\int_{\Omega}|y|^{p_{s}} d x & \leq\left(\int_{\Omega}|y|^{p} d x\right)^{s /(s+1)}|\Omega|^{1 /(s+1)} \leq C\|y\|_{H_{0, B}^{1, p}(\Omega)}^{\frac{p_{s}}{p}} .
\end{aligned}
$$

Hence, each function $y \in H_{0, B}^{1, p}(\Omega)$ belongs to the non-weighted space $W_{0}^{1, p_{s}}(\Omega)$. Combining this fact with the Sobolev embedding theorem, we deduce:

$$
\text { if } s>\frac{N}{p} \text { then } p_{s}^{*}=\frac{N p_{s}}{N-p_{s}}>p,
$$

and, therefore, we have the compact embedding

$$
\begin{gathered}
W_{0}^{1, p_{s}}(\Omega) \hookrightarrow L^{r}(\Omega) \text { and } H_{0, B}^{1, p}(\Omega) \hookrightarrow L^{r}(\Omega), \\
1 \leq r<p_{s}^{*}=\frac{N p s}{(N-p) s+N} .
\end{gathered}
$$


Moreover, as follows from (2.16) and (2.14), the following the weighted Friedrichs inequality

$$
\begin{aligned}
\|y\|_{L^{p}(\Omega)} & \stackrel{\text { by }}{\stackrel{(2.16)}{\leq}} C\|y\|_{W_{0}^{1, p_{s}}(\Omega)}=C\|\nabla y\|_{L^{p_{s}(\Omega)^{N}}} \\
& \stackrel{\text { by }}{\stackrel{(2.14)}{\leq}} C\left\|\alpha^{-1}\right\|_{L^{s+1}(\Omega)}^{1 / p_{s}}\left(\int_{\Omega}|(\nabla y, B(x) \nabla y)|^{p / 2} d x\right)^{1 / p}
\end{aligned}
$$

holds true for each $y \in H_{0, B}^{1, p}(\Omega)$. Hence, the norm

$$
\|y\|_{H_{0, B}^{1, p}(\Omega)}=\left(\int_{\Omega}|(\nabla y, B \nabla y)|^{\frac{p}{2}} d x\right)^{1 / p}
$$

on the space $H_{0, B}^{1, p}(\Omega)$ is equivalent to the norm $\|\cdot\|_{W_{0, B}^{1, p}(\Omega)}$ defined by (2.11).

Weak Convergence in Variable $L^{p}$-Spaces Associated with $\mathbb{S}_{\text {sym }}^{N}$-Matrices. Let $\left\{B_{k}\right\}_{k \in \mathbb{N}}$ and $B$ be a given collection of $\mathbb{S}_{s y m}^{N}$-matrices such that

$$
B_{k}, B \in L^{1}\left(\Omega ; \mathbb{S}_{s y m}^{N}\right) \text { and } B_{k} \rightarrow B \text { in } L^{1}\left(\Omega ; \mathbb{S}_{s y m}^{N}\right)
$$

Let $L^{p}(\Omega, B d x)^{N}$, with $p \geq 2$, be the Lebesgue space of measurable vector-valued functions $f(x) \in \mathbb{R}^{N}$ on $\Omega$ such that

$$
\|f\|_{L^{p}(\Omega, B d x)^{N}}=\left(\int_{\Omega}|(f, B f)|^{\frac{p}{2}} d x\right)^{1 / p}<+\infty .
$$

We say that a sequence $\left\{v_{k} \in L^{p}\left(\Omega, B_{k} d x\right)^{N}\right\}_{k \in \mathbb{N}}$ is bounded if

$$
\limsup _{k \rightarrow \infty} \int_{\Omega}\left|\left(v_{k}, B_{k} v_{k}\right)\right|^{\frac{p}{2}} d x<+\infty .
$$

Definition 2.1. A bounded sequence $\left\{v_{k} \in L^{p}\left(\Omega, B_{k} d x\right)^{N}\right\}_{k \in \mathbb{N}}$ is weakly convergent to a function $v \in L^{p}(\Omega, B d x)^{N}$ in variable space $L^{p}\left(\Omega, B_{k} d x\right)^{N}$ if

$$
\lim _{k \rightarrow \infty} \int_{\Omega}\left(\varphi, B_{k} v_{k}\right) d x=\int_{\Omega}(\varphi, B v) d x \quad \forall \varphi \in C_{0}^{\infty}(\Omega)^{N}
$$

Definition 2.2. A sequence $\left\{v_{k} \in L^{p}\left(\Omega, B_{k} d x\right)^{N}\right\}_{k \in \mathbb{N}}$ is said to be strongly convergent to a function $v \in L^{p}(\Omega, A d x)^{N}$ if

$$
\lim _{k \rightarrow \infty} \int_{\Omega}\left(b_{k}, B_{k} v_{k}\right) d x=\int_{\Omega}(b, B v) d x
$$

whenever $b_{k} \rightarrow b$ in $L^{q}\left(\Omega, B_{k} d x\right)^{N}$ as $k \rightarrow \infty$, where $q=p /(p-1)$ is the Holder conjugate of $p$.

Remark 2.3. Note that in the case $B_{k} \equiv B$, Definitions 2.1-2.2 leads to the wellknown notion of convergence in weighted Lebesgue space $L^{p}(\Omega, B d x)^{N}$. 
The main properties of the weak and strong convergence in $L^{p}\left(\Omega, B_{k} d x\right)^{N}$ can be expressed as follows (see $[17,18]$ for the details):

Proposition 2.1. If a sequence $\left\{v_{k} \in L^{p}\left(\Omega, B_{k} d x\right)^{N}\right\}_{k \in \mathbb{N}}$ is bounded and condition (2.18) holds true, then it is compact with respect to the weak convergence in $L^{p}\left(\Omega, B_{k} d x\right)^{N}$.

Proposition 2.2. If the sequence $\left\{v_{k} \in L^{p}\left(\Omega, B_{k} d x\right)^{N}\right\}_{k \in \mathbb{N}}$ converges weakly to $v \in L^{p}(\Omega, B d x)^{N}$ and condition (2.18) holds true, then

$$
\liminf _{k \rightarrow \infty} \int_{\Omega}\left|\left(v_{k}, B_{k} v_{k}\right)\right|^{\frac{p}{2}} d x \geq \int_{\Omega}|(v, B v)|^{\frac{p}{2}} d x .
$$

Proposition 2.3. Assume the condition (2.18) holds true. Then the weak convergence of a sequence $\left\{v_{k} \in L^{p}\left(\Omega, B_{k} d x\right)^{N}\right\}_{k \in \mathbb{N}}$ to $v \in L^{p}(\Omega, B d x)^{N}$ and

$$
\lim _{k \rightarrow \infty} \int_{\Omega}\left|\left(v_{k}, B_{k} v_{k}\right)\right|^{\frac{p}{2}} d x=\int_{\Omega}|(v, B v)|^{\frac{p}{2}} d x
$$

are equivalent to the strong convergence of $\left\{v_{k}\right\}_{k \in \mathbb{N}}$ to $v$ in $L^{p}\left(\Omega, B_{k} d x\right)^{N}$.

We make also use of the following inequality that was established by Maz'ya in 1972 [23]. If $\mu$ is a positive Radon measure, then

$$
\left(\int_{\Omega}|\varphi|^{r} d \mu\right)^{1 / r} \leq C_{M} \int_{\Omega}|\nabla \varphi| d x \quad \forall \varphi \in C_{0}^{\infty}(\Omega), \forall r \in[1, \infty),
$$

with the best constant

$$
C_{M}=\sup _{\Omega^{\prime} \subset \Omega} \frac{\mu\left(\Omega^{\prime}\right)^{1 / r}}{\mathcal{H}^{N-1}\left(\partial \Omega^{\prime}\right)}
$$

where the supremum in (2.23) is taken over all open subsets of $\Omega$, with $C^{\infty}$-boundary, such that $\overline{\Omega^{\prime}} \subset \Omega$.

\section{Setting of the Boundary Value Problem}

Let $y_{d} \in L^{2}(\Omega)$ and $f \in L^{\infty}(\Omega)^{N}$ be given distributions. For a fixed $A \in \mathfrak{M}_{a d}$, we consider the following boundary value problem:

$$
\begin{gathered}
-\operatorname{div}\left(|(\nabla y, A \nabla y)|^{\frac{p-2}{2}} A \nabla y\right)+|y|^{p-2} y u=-\operatorname{div} f \quad \text { in } \Omega, \\
y=0 \quad \text { on } \partial \Omega, \\
u \in L^{1}(\Omega), \quad u(x) \geq 0 \text { a.e. in } \Omega
\end{gathered}
$$

where we adopt $u$ as a given control function.

It is worth to notice that, in view of the definition of the set $\mathfrak{M}_{a d}$, we deal with a boundary value problem for degenerate quasi-linear elliptic equation with singular coefficients. It means that even for symmetric matrices of coefficients $A \in \mathfrak{M}_{a d}$ 
this problem can exhibit the Lavrentieff phenomenon (i.e. $W_{0, B}^{1, p}(\Omega) \neq H_{0, B}^{1, p}(\Omega)$ ) and, as a consequence, non-uniqueness of the weak solutions. Thus, the original boundary value problem (3.1)-(3.2) is ill-posed, in general.

The another distinguishing feature of the boundary value problem (3.1)(3.2) is the fact that the skew-symmetric part $D$ of the matrix $A \in \mathfrak{M}_{a d}$ is merely measurable and belongs to the space $B M O\left(\Omega ; \mathbb{M}^{N}\right)$ (rather than the space of bounded matrices $\left.L^{\infty}\left(\Omega ; \mathbb{M}^{N}\right)\right)$. This circumstance can entail a number of pathologies with respect to the standard properties of BVPs for elliptic equations with anisotropic $p$-Laplacian even with 'a good' symmetric part $B$ of $A$ and a smooth right-hand side $f$. In particular, the unboundedness of the skew-symmetric part of matrix $A \in \mathfrak{M}_{a d}$ can have a reflection in non-uniqueness of weak solutions to the corresponding boundary value problem. For more details and other types of solutions to elliptic equations with unbounded coefficients we refer to $[7,14-16,33]$.

We associate to the boundary value problem (3.1)-(3.2) the following space $\mathbb{X}_{u, B}=H_{0, B}^{1, p}(\Omega) \cap L^{p}(\Omega, u d x)$. Here, $L^{p}(\Omega, u d x)$ is a usual Banach space with respect to the measure $d \mu=u d x$. Since $u \in L^{1}(\Omega)$ and $u(x) \geq 0$ a.e. in $\Omega$, it follows that $\mu$ is a positive Radon measure and, hence, the space $H_{0, B}^{1, p}(\Omega) \cap$ $L^{p}(\Omega, u d x)$ is well defined and it is a Banach space with respect to the norm (see [3])

$$
\begin{aligned}
\|y\|_{\mathbb{X}_{u, B}} & =\left(\int_{\Omega}|(\nabla y, B \nabla y)|^{\frac{p}{2}} d x+\int_{\Omega}|y|^{p} u d x\right)^{1 / p} \\
& =\left(\|y\|_{H_{0, B}^{1, p}(\Omega)}^{p}+\|y\|_{L^{p}(\Omega, u d x)}^{p}\right)^{1 / p}
\end{aligned}
$$

Definition 3.1. We say that, for a fixed control $u$ and given distributions $A \in$ $\mathfrak{M}_{a d}$, and $f \in L^{\infty}(\Omega)^{N}$, a function $y=y(A, u, f)$ is a weak solution (in the sense of Minty) to boundary value problem (3.1)-(3.2) if $y \in \mathbb{X}_{u, B}$ and the inequality

$$
\begin{aligned}
\int_{\Omega}|(\nabla \varphi, A \nabla \varphi)|^{\frac{p-2}{2}}(A \nabla \varphi, \nabla \varphi-\nabla y) d x+\int_{\Omega}|\varphi|^{p-2} \varphi(\varphi-y) u d x & \\
& \geq \int_{\Omega}(f, \nabla \varphi-\nabla y) d x
\end{aligned}
$$

holds for any $\varphi \in C_{0}^{\infty}(\Omega)$.

To begin with, let us show that this definition makes a sense. Indeed, by the initial assumptions and Hölder's inequality, we have

$$
\begin{aligned}
\int_{\Omega}(f, & \nabla \varphi-\nabla y) d x=\int_{\Omega}\left(\left(L^{-1}\right)^{t} f, L \nabla \varphi-L \nabla y\right) d x \\
& \leq\|f\|_{L^{\infty}(\Omega)^{N}} \int_{\Omega}\left\|L^{-1}\right\||L \nabla \varphi-L \nabla y| d x \\
& \quad \underset{ }{\operatorname{by}(2.9),(2.17)}\|f\|_{L^{\infty}(\Omega)^{N}}\left\|\alpha^{-1}\right\|_{L^{q}(\Omega)}\|\varphi-y\|_{H_{0, B}^{1, p}(\Omega)} \leq C\|\varphi-y\|_{\mathbb{X}_{u, B}}
\end{aligned}
$$


and

$$
\int_{\Omega}|\varphi|^{p-2} \varphi(\varphi-y) u d x \leq\|\varphi\|_{L^{p}(\Omega, u d x)}^{p-1}\|\varphi-y\|_{L^{p}(\Omega, u d x)} \leq C\|\varphi-y\|_{\mathbb{X}_{u, B}} .
$$

As for the first term in (3.4), we observe that

$$
|(\nabla \varphi, A \nabla \varphi)|^{\frac{p-2}{2}}=\left|\left(L \nabla \varphi,[\underbrace{I+\left(L^{t}\right)^{-1} D L^{-1}}_{T}] L \nabla \varphi\right)\right|^{\frac{p-2}{2}} \leq\|T\|^{\frac{p-2}{2}}|L \nabla \varphi|^{p-2}
$$

and, therefore,

$$
\begin{aligned}
\int_{\Omega}|(\nabla \varphi, A \nabla \varphi)|^{\frac{p-2}{2}} & (A \nabla \varphi, \nabla \varphi-\nabla y) d x \\
& \leq \int_{\Omega}\|T\|^{\frac{p-2}{2}}|L \nabla \varphi|^{p-2}(T L \nabla \varphi, L \nabla \varphi-L \nabla y) d x \\
& \leq \int_{\Omega}\|T\|^{\frac{p}{2}}|L \nabla \varphi|^{p-1}|L \nabla \varphi-L \nabla y| d x \\
& \leq\|\varphi\|_{C^{1}(\Omega)}^{p-1} \int_{\Omega}\|T\|^{\frac{p}{2}} \beta^{p-1}|L \nabla \varphi-L \nabla y| d x \\
& \leq\|\varphi\|_{C^{1}(\Omega)}^{p-1}\left(\int_{\Omega}\|T\|^{\frac{p q}{2}} \beta^{p} d x\right)^{1 / q}\|\varphi-y\|_{H_{0, B}^{1, p}(\Omega)} .
\end{aligned}
$$

Since,

$$
\begin{aligned}
\int_{\Omega}\|T\|^{\frac{p q}{2}} \beta^{p} d x & \leq \int_{\Omega}\left(1+\alpha^{-2}\|D\|\right)^{\frac{p q}{2}} \beta^{p} d x \\
& \leq 2^{p q-1} \int_{\Omega}\left(\beta^{p}+\left(\alpha^{-q} \beta\right)^{p}\|D\|^{\frac{p q}{2}}\right) d x \\
& \leq 2^{p q-1}\left[\|\beta\|_{L^{p}(\Omega)}^{p}+\left\|\alpha^{-1}\right\|_{L^{4 p q}(\Omega)}^{p q}\|\beta\|_{L^{4 p}(\Omega)}^{p}\|D\|_{L^{p q}\left(\Omega ; \mathbb{S}_{s k e w}^{N}\right)}^{\frac{p q}{2}}\right] \\
& \stackrel{\text { by }(2.4)}{<}+\infty
\end{aligned}
$$

it follows from (3.7) that

$$
\int_{\Omega}|(\nabla \varphi, A \nabla \varphi)|^{\frac{p-2}{2}}(A \nabla \varphi, \nabla \varphi-\nabla y) d x \leq C\|\varphi-y\|_{\mathbb{X}_{u, B}}
$$

Thus, the well posedness of each term in the variational inequality (3.4) and, hence, the consistency of the definition of the weak solution in the sense of Minty to the considered boundary value problem, obviously follows from the estimates (3.5)-(3.6), (3.8).

Remark 3.1. The estimate (3.8) and the fact that $(\nabla \varphi(x), D(x) \nabla \varphi(x))=0$ a.e. in $\Omega$ by the skew-symmetry property of $D$, imply that the variational inequality 
(3.4) can be rewritten as follows

$$
\begin{aligned}
\int_{\Omega}|(\nabla \varphi, B \nabla \varphi)|^{\frac{p-2}{2}}(A \nabla \varphi, \nabla \varphi-\nabla y) d x+\int_{\Omega}|\varphi|^{p-2} \varphi(\varphi-y) u d x & \\
& \geq \int_{\Omega}(f, \nabla \varphi-\nabla y) d x .
\end{aligned}
$$

Getting inspired by this, we call a function $y \in \mathbb{X}_{u, B}$ a weak solution (in the sense of Minty) to boundary value problem (3.1)-(3.2) if it satisfies the inequality (3.9) for every test function $\varphi \in C_{0}^{\infty}(\Omega)$.

Taking this remark into account, it is reasonable to consider another definition of the weak solution to the given boundary value problem, in the terms of distributions, which appears more natural:

$$
\begin{aligned}
& y \in \mathbb{X}_{u, B} \text { is the distributional solution to (3.1)-(3.2) if the integral identity } \\
& \int_{\Omega}|(\nabla y, B \nabla y)|^{\frac{p-2}{2}}(A \nabla y, \nabla \varphi) d x+\int_{\Omega}|y|^{p-2} y \varphi u d x=\int_{\Omega}(f, \nabla \varphi) d x
\end{aligned}
$$

holds true for every $\varphi \in C_{0}^{\infty}(\Omega)$.

In spite of the fact that the relations between these definitions are very intricate for general matrix $A \in \mathfrak{M}_{a d}$ (for an example when these definitions lead to the different solutions even for linear equations, we refer to [25]), we can leverage the integral identity (3.10) for the following estimate

$$
\begin{aligned}
& \left.\left|\int_{\Omega}\right|(\nabla y, B \nabla y)\right|^{\frac{p-2}{2}}(A \nabla y, \nabla \varphi) d x \mid \\
& \quad \leq \int_{\Omega}|y|^{p-1} u^{\frac{p-1}{p}}|\varphi| u^{\frac{1}{p}} d x+\int_{\Omega}\left|\left(L^{-1}\right)^{t} f\right||L \nabla \varphi| d x \\
& \quad \leq\|y\|_{L^{p}(\Omega, u d x)}^{p-1}\|\varphi\|_{L^{p}(\Omega, u d x)}+\|f\|_{L^{\infty}(\Omega)^{N}}\left\|\alpha^{-1}\right\|_{L^{q}(\Omega)}\|\varphi\|_{H_{0, B}^{1, p}(\Omega)} \\
& \quad \leq\left[\|y\|_{L^{p}(\Omega, u d x)}^{p-1}+\|f\|_{L^{\infty}(\Omega)^{N}}\left\|\alpha^{-1}\right\|_{L^{q}(\Omega)}\right]\|\varphi\|_{\mathbb{X}_{u, B}} \\
& \quad=C(y, u, B, f)\|\varphi\|_{\mathbb{X}_{u, B} .}
\end{aligned}
$$

Remark 3.2. As follows from (3.11), a weak solution to the considered problem in the sense of distribution belongs to the special subset $D\left(\mathbb{X}_{u, B}\right)$ of the space $\mathbb{X}_{u, B}:=H_{0, B}^{1, p}(\Omega) \cap L^{p}(\Omega, u d x)$, elements of which possess the property (3.11). As a result, if $y \in D\left(\mathbb{X}_{u, B}\right)$ then the mapping

$$
\varphi \mapsto[y, \varphi]_{A}:=\int_{\Omega}|(\nabla y, B \nabla y)|^{\frac{p-2}{2}}(A \nabla y, \nabla \varphi) d x
$$

can be defined for all test functions $\varphi \in \mathbb{X}_{u, B}$ using the standard rule

$$
[y, z]_{A}=\lim _{k \rightarrow \infty}\left[y, \varphi_{k}\right]_{A}
$$


where $\left\{\varphi_{k}\right\}_{k \in \mathbb{N}} \subset C_{0}^{\infty}(\Omega)$ and $\varphi_{k} \rightarrow z$ strongly in $\mathbb{X}_{u, B}$ (it is the case when we essentially use the fact that $C_{0}^{\infty}(\Omega)$ is dense in $\left.H_{0, B}^{1, p}(\Omega) \cap L^{p}(\Omega, u d x)\right)$. In particular, if $y \in D\left(\mathbb{X}_{u, B}\right)$, then we can define the value $[y, y]_{A}$ and this one is finite for every $y \in D\left(\mathbb{X}_{u, B}\right)$, although the "integrand"

$$
|(\nabla y, B \nabla y)|^{\frac{p}{2}}+|(\nabla y, B \nabla y)|^{\frac{p-2}{2}}(D \nabla y, \nabla y)
$$

needs not be integrable on $\Omega$, in general. As a result, we can derive form (3.10) the energy equality for distributional solutions

$$
[y, y]_{A}+\int_{\Omega}|y|^{p} u d x=\int_{\Omega}(f, \nabla y) d x .
$$

However, as it follows from definition of the form $[y, \varphi]_{A}$, the value $[y, y]_{A}$ is not equal to $\|y\|_{H_{0, B}^{1, p}(\Omega)}^{p}$, in general, and it does not preserve the inequality

$$
[y, y]_{A} \geq\|y\|_{H_{0, B}^{1, p}(\Omega)}^{p} \quad \text { for all } y \in D\left(\mathbb{X}_{u, B}\right) .
$$

Hence, even if the relation $H_{0, B}^{1, p}(\Omega)=W_{0, B}^{1, p}(\Omega)$ holds true, the energy equality (3.12) does not allow us to derive a reasonable a priory estimate in $\|\cdot\|_{\mathbb{X}_{u, B}}$-norm for the weak solutions in the sense of distributions.

\section{On Solvability of Boundary Value Problem (3.1)-(3.3)}

Our main intension in this section is to show that boundary value problem admits a weak solution due to the approximation approach. It is clear that the condition $A \in \mathfrak{M}_{a d}(\Omega)$ ensures the existence of the sequence of matrices $\left\{A_{k}\right\}_{k \in \mathbb{N}} \subset \mathfrak{M}_{a d}(\Omega) \cap L^{\infty}\left(\Omega ; \mathbb{M}^{N}\right)$ such that $A_{k} \rightarrow A$ strongly in $L^{1}\left(\Omega ; \mathbb{M}^{N}\right)$. With that in mind we give a few auxiliary results.

Lemma 4.1. Let $\left\{A_{k}\right\}_{k \in \mathbb{N}} \subset \mathfrak{M}_{a d}(\Omega)$ and $A \in \mathfrak{M}_{a d}(\Omega)$ be matrices such that

$$
\begin{gathered}
A_{k} \in L^{\infty}\left(\Omega ; \mathbb{M}^{N}\right) \quad \forall k \in \mathbb{N}, \\
A_{k} \rightarrow A \quad \text { strongly in } L^{1}\left(\Omega ; \mathbb{M}^{N}\right), \\
\left(\eta, A_{k} \eta\right) \geq \alpha_{k}^{2}|\eta|^{2} \text { a.e. in } \Omega \forall \eta \in \mathbb{R}^{N} \\
\text { and for some positive } \alpha_{k} \in \mathbb{R}, \alpha_{k} \geq \alpha(x) .
\end{gathered}
$$

Then

$$
L_{k}^{-1} \rightarrow L^{-1} \quad \text { and } \quad T_{k} \rightarrow T \text { strongly in } L^{1}\left(\Omega ; \mathbb{M}^{N}\right),
$$

where

$$
\begin{aligned}
B_{k}:=\frac{1}{2}\left(A_{k}+A_{k}^{t}\right)=L_{k}^{t} L_{k}, B & :=\frac{1}{2}\left(A+A^{t}\right)=L^{t} L, \\
T_{k} & :=I+\left(L_{k}^{t}\right)^{-1} D_{k} L_{k}^{-1}, T:=I+\left(L^{t}\right)^{-1} D L^{-1}, \\
D_{k} & :=\frac{1}{2}\left(A_{k}-A_{k}^{t}\right), D:=\frac{1}{2}\left(A-A^{t}\right) .
\end{aligned}
$$


Remark 4.1. The simplest way to construct a sequence $\left\{A_{k}\right\}_{k \in \mathbb{N}} \subset \mathfrak{M}_{a d}(\Omega)$, possessing the properties (4.1)-(4.3), is to set

$$
A_{k}=k^{-1} I+\left[\max \left\{\min \left\{a_{i j}, k\right\},-k\right\}\right]_{i, j=1}^{N}
$$

or apply the procedure of the direct Steklov smoothing to a given matrix $A \in$ $\mathfrak{M}_{a d}(\Omega)$ with some positive compactly supported smooth kernel (see, for instance, $[15])$.

Proof. The conditions (4.1)-(4.3) ensure that $B_{k}^{-1} \in L^{\infty}\left(\Omega ; \mathbb{S}_{\text {sym }}^{N}\right)$ for all $k \in \mathbb{N}$ and (up to a subsequence)

$$
D_{k}(x) \rightarrow D(x) \text { and } L_{k}^{-1}(x) \rightarrow L^{-1}(x) \text { a.e. in } \Omega .
$$

Moreover, since $\alpha_{k} \geq \alpha$ a.e. in $\Omega$, it follows that

$$
\left\|L_{k}^{-1}(x)\right\| \leq \alpha_{k}^{-1} \leq \alpha^{-1}(x) \text { a.e. in } \Omega,
$$

where $\alpha^{-1} \in L^{1}(\Omega)$ (see (2.2)). Hence, the sequence $\left\{L_{k}^{-1}\right\}_{k \in \mathbb{N}}$ is equi-integrable. In view of the definition of the class $\mathfrak{M}_{a d}(\Omega)$, the same conclusion can be made for the sequence of skew-symmetric matrices $\left\{\left(L_{k}^{t}\right)^{-1} D_{k} L_{k}^{-1}\right\}_{k \in \mathbb{N}}$. As a result, the property (4.4) is a direct consequence of Lebesgue's Theorem.

Lemma 4.2. Let $f \in L^{\infty}(\Omega)^{N}$ be a given distribution, and let $\left\{A_{k}\right\}_{k \in \mathbb{N}} \subset \mathfrak{M}_{a d}(\Omega)$ and $A \in \mathfrak{M}_{a d}(\Omega)$ be matrices satisfying the properties (4.1)-(4.3). Then, for an arbitrary smooth function $\varphi \in C_{0}^{\infty}(\Omega)$, the sequences

$$
\left\{v_{k}:=\left|\left(\nabla \varphi, B_{k} \nabla \varphi\right)\right|^{\frac{p-2}{2}} L_{k}^{-1} T_{k} L_{k} \nabla \varphi\right\}_{k \in \mathbb{N}} \text { and } \quad\left\{w_{k}:=B_{k}^{-1} f\right\}_{k \in \mathbb{N}}
$$

are bounded in $L^{q}\left(\Omega, B_{k} d x\right)^{N}$ and

$$
\begin{gathered}
v_{k} \rightarrow v=|(\nabla \varphi, B \nabla \varphi)|^{\frac{p-2}{2}} L^{-1} T L \nabla \varphi \text { strongly in variable } L^{q}\left(\Omega, B_{k} d x\right)^{N}, \\
w_{k} \rightarrow w=B^{-1} f \text { strongly in variable } L^{q}\left(\Omega, B_{k} d x\right)^{N},
\end{gathered}
$$

where the matrices $T_{k}$ and $T$ are defined by (4.5).

Proof. Indeed, by definition of the space $L^{q}\left(\Omega, B_{k} d x\right)^{N}$, we have

$$
\begin{aligned}
& \left\|v_{k}\right\|_{L^{q}\left(\Omega, B_{k} d x\right)^{N}}^{q}=\int_{\Omega}\left|\left(v_{k}, B_{k} v_{k}\right)\right|^{\frac{q}{2}} d x=\int_{\Omega}\left|L_{k} v_{k}\right|^{q} d x \\
& \quad=\left.\left.\int_{\Omega}||\left(\nabla \varphi, B_{k} \nabla \varphi\right)\right|^{\frac{p-2}{2}} T_{k} L_{k} \nabla \varphi\right|^{q} d x \leq\|\varphi\|_{C^{1}(\Omega)}^{p} \int_{\Omega}\left[\left\|L_{k}\right\|^{p-1}\left\|T_{k}\right\|\right]^{q} d x \\
& \quad \leq\|\varphi\|_{C^{1}(\Omega)}^{p} \int_{\Omega}\left[\beta^{p-1}\left(1+\alpha^{-2}\left\|D_{k}\right\|\right) \|\right]^{q} d x \\
& \quad \leq 2^{q-1}\|\varphi\|_{C^{1}(\Omega)}^{p} \int_{\Omega} \beta^{p}\left(1+\alpha^{-2 q}\left\|D_{k}\right\|^{q}\right) d x \\
& \quad \leq 2^{q-1}\|\varphi\|_{C^{1}(\Omega)}^{p}\left[\|\beta\|_{L^{p}(\Omega)}^{p}+\|\beta\|_{L^{3 p}(\Omega)}^{p}\left\|\alpha^{-1}\right\|_{L^{6 q}(\Omega)}^{2 q}\|D\|_{L^{3 q}(\Omega)}^{q}\right] \\
& \quad \text { by (2.4) } \quad \leq \text { const }<+\infty .
\end{aligned}
$$


Hence, the sequence $\left\{v_{k}\right\}_{k \in \mathbb{N}}$ is bounded in $L^{q}\left(\Omega, B_{k} d x\right)^{N}$.

Further we notice that, by the initial assumption (4.2), Lemma 4.1, and BMOproperties of the matrices $L, L^{-1}$, and $D$, we see that the sequence

$$
\left\{\left|\left(\nabla \varphi, B_{k} \nabla \varphi\right)\right|^{\frac{p-2}{2}} T_{k} L_{k} \nabla \varphi\right\}_{k \in \mathbb{N}}
$$

is equi-integrable and

$$
\left|\left(\nabla \varphi, B_{k} \nabla \varphi\right)\right|^{\frac{p-2}{2}} T_{k} L_{k} \nabla \varphi \rightarrow|(\nabla \varphi, B \nabla \varphi)|^{\frac{p-2}{2}} T L \nabla \varphi \quad \text { a.e. in } \Omega
$$

for any $\varphi \in C_{0}^{\infty}(\Omega)$. Hence, by Lebesgue's Theorem, we have the strong convergence

$$
\left|\left(\nabla \varphi, B_{k} \nabla \varphi\right)\right|^{\frac{p-2}{2}} T_{k} L_{k} \nabla \varphi \rightarrow|(\nabla \varphi, B \nabla \varphi)|^{\frac{p-2}{2}} T L \nabla \varphi \quad \text { in } \quad L^{1}\left(\Omega ; \mathbb{R}^{N}\right) .
$$

As a result, this implies

$$
\begin{aligned}
\lim _{k \rightarrow \infty} \int_{\Omega}\left(\nabla \psi, B_{k} v_{k}\right) d x & =\lim _{k \rightarrow \infty} \int_{\Omega}\left|\left(\nabla \varphi, B_{k} \nabla \varphi\right)\right|^{\frac{p-2}{2}}\left(\nabla \psi, T_{k} L_{k} \nabla \varphi\right) d x \\
& \stackrel{\text { by }}{=} \int_{\Omega}|(\nabla \varphi, B \nabla \varphi)|^{\frac{p-2}{2}}(\nabla \psi, T L \nabla \varphi) d x \\
& =\int_{\Omega}(\nabla \psi, B v) d x, \quad \forall \psi \in C_{0}^{\infty}(\Omega) .
\end{aligned}
$$

Thus, the sequence $\left\{v_{k}\right\}_{k \in \mathbb{N}}$ is weakly convergent in $L^{q}\left(\Omega, B_{k} d x\right)^{N}$ to the vector-valued function $v=|(\nabla \varphi, B \nabla \varphi)|^{\frac{p-2}{2}} L^{-1} T L \nabla \varphi$.

It remains to show that the sequence $\left\{v_{k}\right\}_{k \in \mathbb{N}}$ is strongly convergent to $v$. To do so, we make use of Proposition 2.3. Following this assertion, it is enough to prove the equality

$$
\begin{aligned}
\lim _{k \rightarrow \infty} \int_{\Omega} \mid & \left.\left(v_{k}, B_{k} v_{k}\right)\right|^{\frac{q}{2}} d x=\lim _{k \rightarrow \infty} \int_{\Omega}\left|L_{k} v_{k}\right|^{q} d x \\
& =\left.\left.\lim _{k \rightarrow \infty} \int_{\Omega}||\left(\nabla \varphi, B_{k} \nabla \varphi\right)\right|^{\frac{p-2}{2}} T_{k} L_{k} \nabla \varphi\right|^{q} d x \\
& =\left.\left.\int_{\Omega}||(\nabla \varphi, B \nabla \varphi)\right|^{\frac{p-2}{2}} T L \nabla \varphi\right|^{q} d x=\int_{\Omega}(v, B v)^{\frac{q}{2}} d x .
\end{aligned}
$$

In view of the estimate

$$
\left.\left.||\left(\nabla \varphi, B_{k} \nabla \varphi\right)\right|^{\frac{p-2}{2}} T_{k} L_{k} \nabla \varphi\left|\leq\left\|L_{k} \nabla \varphi\right\|^{p-1}\left\|T_{k}\right\| \leq \beta^{p-1}\|T\|\right| \nabla \varphi\right|^{p-1}
$$

and the fact that the term $\left(\beta^{p-1}\|T\||\nabla \varphi|^{p-1}\right)^{q}=\beta^{p}\|T\|^{q}|\nabla \varphi|^{p}$ is in $L^{1}(\Omega)$ by Remark 2.1, we see that the sequence $\left\{\left|\left(v_{k}, B_{k} v_{k}\right)\right|^{\frac{q}{2}}\right\}_{k \in \mathbb{N}}$ is equi-integrable. On the other hand, property (4.2) and Lemma 4.1 imply that, within a subsequence,

$$
\left|\left(\nabla \varphi, B_{k} \nabla \varphi\right)\right|^{\frac{p-2}{2}} T_{k} L_{k} \rightarrow|(\nabla \varphi, B \nabla \varphi)|^{\frac{p-2}{2}} T L \text { almost everywhere in } \Omega .
$$


Therefore, the equality (4.11) is a direct consequence of Lebesgue Dominated Theorem. Thus, the strong convergence in variable space $L^{q}\left(\Omega, B_{k} d x\right)^{N}$ of the sequence $\left\{v_{k}\right\}_{k \in \mathbb{N}}$ is established.

The property (4.7) can be proved following the same arguments.

For our further analysis, we make use of the following concept.

Definition 4.1. We say that a bounded sequence

$$
\left\{\left(A_{k}, y_{k}\right) \in \mathfrak{M}_{a d}(\Omega) \times\left[H_{0, B_{k}}^{1, p}(\Omega) \cap L^{p}(\Omega, u d x)\right]\right\}_{k \in \mathbb{N}}
$$

$w$-converges to the pair $(A, y) \in \mathfrak{M}_{a d}(\Omega) \times\left[H_{0, B}^{1, p}(\Omega) \cap L^{p}(\Omega, u d x)\right]$ as $k \rightarrow \infty$ (in symbols, $\left.\left(A_{k}, y_{k}\right) \stackrel{w}{\longrightarrow}(A, y)\right)$ if

$$
A_{k} \rightarrow A \quad \text { in } L^{1}\left(\Omega ; \mathbb{M}^{N}\right),
$$

$y_{k} \rightarrow y \quad$ in $L^{p}(\Omega)$ and weakly in weighted space $L^{p}(\Omega, u d x)$,

$\nabla y_{k} \rightarrow \nabla y \quad$ in the variable space $L^{p}\left(\Omega, B_{k} d x\right)^{N}$.

In particular, as follows from this definition, if $\left(A_{k}, y_{k}\right) \stackrel{w}{\rightarrow}(A, y)$, then

$$
\begin{gathered}
\lim _{k \rightarrow \infty} \int_{\Omega}\left\|A_{k}\right\| d x=\int_{\Omega}\|A\| d x, \\
\lim _{k \rightarrow \infty} \int_{\Omega} y_{k} \varphi u d x=\int_{\Omega} y \varphi u d x \quad \forall \varphi \in C_{0}^{\infty}(\Omega), \\
\lim _{k \rightarrow \infty} \int_{\Omega}\left(\xi, B_{k} \nabla y_{k}\right) d x=\int_{\Omega}(\xi, B \nabla y) d x \quad \forall \xi \in C_{0}^{\infty}(\Omega)^{N} .
\end{gathered}
$$

In order to motivate this definition, we give the following result.

Lemma 4.3. Let $\left\{\left(A_{k}, y_{k}\right) \in \mathfrak{M}_{a d}(\Omega) \times\left[H_{0, B_{k}}^{1, p}(\Omega) \cap L^{p}(\Omega, u d x)\right]\right\}_{k \in \mathbb{N}}$ be a sequence with the following properties:

(i) $A_{k} \in L^{\infty}\left(\Omega ; \mathbb{M}^{N}\right) \forall k \in \mathbb{N}$, and there exists a matrix $A \in \mathfrak{M}_{a d}(\Omega)$ such that $A_{k} \rightarrow A$ in $L^{1}\left(\Omega ; \mathbb{M}^{N}\right)$

(ii) $\left\{y_{k} \in H_{0, B_{k}}^{1, p}(\Omega) \cap L^{p}(\Omega, u d x)\right\}_{k \in \mathbb{N}}$ are bounded sequences, i.e.

$$
\sup _{k \in \mathbb{N}} \int_{\Omega}\left(u\left|y_{k}\right|^{p}+\left(\nabla y_{k}, B_{k} \nabla y_{k}\right)^{\frac{p}{2}}\right) d x<+\infty
$$

Then, within a subsequence, the original sequence is $w$-convergent. Moreover, each w-limit pair $(A, y)$ belongs to the set $\mathfrak{M}_{a d}(\Omega) \times\left[H_{0, B}^{1, p}(\Omega) \cap L^{p}(\Omega, u d x)\right]$. 
Proof. To begin with, we note that the conditions (i)-(ii) and estimates (2.12)(2.13) immediately imply the boundedness of the sequence

$$
\left\{y_{k} \in H_{0, B}^{1, p}(\Omega) \cap L^{p}(\Omega, u d x)\right\}_{k \in \mathbb{N}}
$$

in $W^{1,1}\left(\Omega ; \mathbb{M}^{N}\right)$ and in variable spaces $H_{0, B_{k}}^{1, p}(\Omega)$ and $L^{p}(\Omega, u d x)$. Moreover, due to the inequalities (2.14)-(2.15), we have the compact embedding

$$
H_{0, B_{k}}^{1, p}(\Omega) \hookrightarrow L^{r}(\Omega) \text { for all } 1 \leq r<p_{s}^{*}=\frac{N p s}{(N-p) s+N} .
$$

Since $p_{s}^{*}=\frac{N p_{s}}{N-p_{s}}>p$ provided $s>\frac{N}{p}$, it follows that the sequence $\left\{y_{k}\right\}_{k \in \mathbb{N}}$ is compact with respect to the norm topology of $L^{p}(\Omega)$.

Thus, combining this fact with the compactness criterium for the weak convergence in variable spaces (see Proposition 2.1), we can deduce the existence of a pair $(y, z) \in L^{p}(\Omega) \times L^{p}(\Omega, u d x) \times L^{p}(\Omega, B d x)^{N}$ such that, within a subsequence of $\left\{y_{k}\right\}_{k \in \mathbb{N}}$, we have

$$
\begin{gathered}
y_{k} \rightarrow y \text { in } L^{p}(\Omega), \\
y_{k} \rightarrow z \text { in } L^{p}(\Omega, u d x), \\
\nabla y_{k} \rightarrow v \text { in the variable space } L^{p}\left(\Omega, B_{k} d x\right)^{N} .
\end{gathered}
$$

Our aim is to show that $y=z, v=\nabla y$, and as a consequence $y \in H_{0, B}^{1, p}(\Omega) \cap$ $L^{p}(\Omega, u d x)$. With that in mind, we note that for every measurable subset $K \subset \Omega$, the estimate

$$
\begin{aligned}
\int_{K}\left|\nabla y_{k}\right| d x & \leq\left(\int_{K}\left|L_{k} \nabla y_{k}\right|^{p} d x\right)^{\frac{1}{p}}\left(\int_{K} \alpha^{-q} d x\right)^{\frac{1}{q}} \\
& \leq\left(\int_{\Omega}\left|\left(\nabla y_{k}, B_{k} \nabla y_{k}\right)\right|^{\frac{p}{2}} d x\right)^{\frac{1}{p}}\left(\int_{K} \alpha^{-q} d x\right)^{\frac{1}{q}} \\
& \text { by } \stackrel{(4.13)}{\leq} C|K|^{\frac{1}{2 q}}\left\|\alpha^{-1}\right\|_{L^{2 q}(\Omega)} \\
& \stackrel{\text { by }}{\leq} C_{1}|K|^{\frac{1}{2 q}}\left(\|\alpha\|_{L^{1}(\Omega)}^{2 q}+\left\|\alpha^{-1}\right\|_{B M O\left(\mathbb{R}^{N}\right)}\right)^{\frac{1}{2 q}}
\end{aligned}
$$

implies equi-integrability of the family $\left\{\left|\nabla y_{k}\right|_{\mathbb{R}^{N}}\right\}$. Combining this fact with estimate (2.13) and property (ii), we deduce that the sequence $\left\{\left|\nabla y_{k}\right|\right\}_{k \in \mathbb{N}}$ is weakly compact in $L^{1}(\Omega)$. Since, for an arbitrary $\xi \in C_{0}^{\infty}(\Omega)^{N}$, we have

$$
B_{k}^{-1} \xi \rightarrow B^{-1} \xi \text { strongly in variable } L^{q}\left(\Omega, B_{k} d x\right)^{N}
$$

by Lemma 4.2 , it follows that

$$
\begin{aligned}
\int_{\Omega}\left(\xi, \nabla y_{k}\right) d x & =\int_{\Omega}\left(B_{k}^{-1} \xi, B_{k} \nabla y_{k}\right) d x \\
\text { by }(4.16),(\stackrel{4.17)}{\longrightarrow}, \text { and }(2.20) & \int_{\Omega}\left(B^{-1} \xi, B v\right) d x \\
& =\int_{\Omega}(\xi, v) d x \quad \forall \xi \in C_{0}^{\infty}(\Omega)^{N} .
\end{aligned}
$$


Thus, in view of the weak compactness property of $\left\{\nabla y_{k}\right\}_{k \in \mathbb{N}}$ in $L^{1}(\Omega)^{N}$, we conclude

$$
\nabla y_{k} \rightarrow v \text { in } L^{1}\left(\Omega ; \mathbb{R}^{N}\right) \quad \text { as } n \rightarrow \infty .
$$

Since $y_{k} \in W^{1,1}(\Omega)$ for all $k \in \mathbb{N}$ and the Sobolev space $W^{1,1}(\Omega)$ is complete, (4.14) and (4.18) imply $\nabla y=v$, and consequently $y \in H_{0, B}^{1, p}(\Omega)$.

To end the proof, it remains to establish the equality $y=z$ a.e. in $\Omega$. Since the sequence $\left\{y_{k} \in L^{p}(\Omega, u d x)\right\}_{k \in \mathbb{N}}$ is bounded and for any measurable set $K \subseteq \Omega$, we have

$$
\int_{K} y_{k} u d x \leq\left(\int_{\Omega}|y|^{p} u d x\right)^{1 / p}\left(\int_{K} u d x\right)^{1 / q},
$$

it follows that the sequence $\left\{y_{k} u\right\}_{k \in \mathbb{N}}$ is equi-integrable and weakly compact in $L^{1}(\Omega)$ and, hence, the weak convergence (4.15) is equivalent to the weak convergence

$$
y_{k} u \rightarrow z u \text { in } L^{1}(\Omega) \text {. }
$$

Further, we note that

$$
\begin{aligned}
\int_{\Omega}|\varphi| u d x & \leq \sup _{\Omega^{\prime} \subset \Omega} \frac{\int_{\Omega^{\prime}}|u| d x}{\mathcal{H}^{N-1}\left(\partial \Omega^{\prime}\right)} \int_{\Omega}|\nabla \varphi| d x \\
& \leq \sup _{\Omega^{\prime} \subset \Omega} \frac{\|u\|_{L^{1}\left(\Omega^{\prime}\right)}}{\mathcal{H}^{N-1}\left(\partial \Omega^{\prime}\right)}\left(\int_{\Omega}|L \nabla \varphi|^{p} d x\right)^{1 / p}\left(\int_{\Omega} \alpha^{-q} d x\right)^{1 / q} \\
& \leq \mathrm{const}\|\varphi\|_{H_{0, B}^{1, p}(\Omega)} \quad \forall \varphi \in C_{0}^{\infty}(\Omega)
\end{aligned}
$$

by Maz'ya inequality (2.23). Since the set $C_{0}^{\infty}(\Omega)$ is dense in $H_{0, B}^{1, p}(\Omega)$, it follows that the family $\left\{u\left(y_{k}-y\right)\right\}_{k \in \mathbb{N}}$ is weakly compact in $L^{1}(\Omega)$. Taking into account the compactness of the embedding $H_{0, B}^{1, p}(\Omega) \hookrightarrow L^{p}(\Omega)$ and the weak convergence $y_{k} \rightarrow y$ in $L^{p}(\Omega)$, we can suppose that $y_{k} \rightarrow y$ almost everywhere in $\Omega$. Hence, $u\left(y_{k}-y\right) \rightarrow 0$ a.e. in $\Omega$. Then the strong convergence $u\left(y_{k}-y\right) \rightarrow 0$ in $L^{1}(\Omega)$ immediately follows from the Lebesgue Theorem. Thus, in order to conclude the desired equality $y=z$, it is enough to combine this inference with the property (4.19). The proof is complete.

We are now in a position to prove the main result of this section. Namely, we show that the boundary value problem (3.1)-(3.3) admits a weak solution.

Theorem 4.1. For given $f \in L^{\infty}(\Omega)^{N}, u \in L^{1}(\Omega), u \geq 0$ a.e. in $\Omega, \gamma>0$, and for an arbitrary matrix $A \in \mathfrak{M}_{a d}$, there exists a weak solution $y \in \mathbb{X}_{u, B}$ (in the sense of Minty) to boundary value problem (3.1)-(3.2) with an a priori estimate

$$
\|y\|_{\mathbb{X}_{u, B}} \leq\left(C_{Q, q}\|f\|_{L^{\infty}(\Omega)^{N}}\right)^{\frac{1}{p-1}}\left(\left\|\alpha^{-1}\right\|_{B M O\left(\mathbb{R}^{N}\right)}+\left\|\alpha^{-1}\right\|_{L^{1}(\Omega)}^{q}\right)^{\frac{1}{p}}
$$

and the energy relation

$$
\int_{\Omega}|(\nabla y, B \nabla y)|^{\frac{p}{2}} d x+\int_{\Omega}|y|^{p} u d x \leq \int_{\Omega}(f, \nabla y) d x .
$$


Proof. Let $u \in \mathfrak{U}_{a d}$ be an arbitrary admissible control. For a given matrix $A \in \mathfrak{M}_{a d}$ let us consider an approximation $\left\{A_{k}\right\}_{k \in \mathbb{N}} \subset \mathfrak{M}_{a d}(\Omega)$ with properties (4.1)-(4.3), and the corresponding variational problem

$$
\begin{aligned}
& \text { Find } y_{k} \in W_{0}^{1, p}(\Omega) \text { such that } \\
& \int_{\Omega}\left|\left(\nabla y_{k}, A_{k} \nabla y_{k}\right)\right|^{\frac{p-2}{2}}\left(A_{k} \nabla y_{k}, \nabla \varphi\right) d x+\int_{\Omega}\left|y_{k}\right|^{p-2} y_{k} \varphi u d x \\
& =\int_{\Omega}(f, \nabla \varphi) d x, \quad \forall \varphi \in C_{0}^{\infty}(\Omega) .
\end{aligned}
$$

Since $A_{k} \in L^{\infty}\left(\Omega ; \mathbb{M}^{N}\right)$, it follows that $\left(\nabla y_{k}, A_{k} \nabla y_{k}\right)=\left(\nabla y_{k}, B_{k} \nabla y_{k}\right)$. Hence, by the well-known result of quasi-linear elliptic equations (see [29, Theorem 2.14]), for every $k \in \mathbb{N}$, the problem (4.22) admits a unique weak solution $y_{k} \in W_{0}^{1, p}(\Omega)$ such that

$$
\int_{\Omega}\left|\left(\nabla y_{k}, B_{k} \nabla y_{k}\right)\right|^{\frac{p}{2}} d x+\int_{\Omega}\left|y_{k}\right|^{p} u d x=\int_{\Omega}\left(f, \nabla y_{k}\right) d x
$$

and

$$
\begin{gathered}
\int_{\Omega}\left|\left(\nabla \varphi, B_{k} \nabla \varphi\right)\right|^{\frac{p-2}{2}}\left(A_{k} \nabla \varphi, \nabla \varphi-\nabla y_{k}\right) d x+\int_{\Omega}|\varphi|^{p-2} \varphi\left(\varphi-y_{k}\right) u d x \\
\geq \int_{\Omega}\left(f, \nabla \varphi-\nabla y_{k}\right) d x, \quad \forall \varphi \in C_{0}^{\infty}(\Omega) .
\end{gathered}
$$

It is clear that the energy equality (4.23) leads to the following estimate

$$
\begin{aligned}
\left\|y_{k}\right\|_{\mathbb{X}_{u, B_{k}}}^{p} & :=\int_{\Omega}\left|\left(\nabla y_{k}, B_{k} \nabla y_{k}\right)\right|^{\frac{p}{2}} d x+\int_{\Omega}\left|y_{k}\right|^{p} u d x \leq \int_{\Omega}\left|\left(L_{k}^{-1}\right)^{t} f\right|\left|L_{k} \nabla y_{k}\right| d x \\
& \leq\|f\|_{L^{\infty}(\Omega)^{N}}\left\|\alpha^{-1}\right\|_{L^{q}(\Omega)}\left\|y_{k}\right\|_{H_{0, B_{k}}^{1, p}(\Omega)} \\
& \leq C_{Q, q}\|f\|_{L^{\infty}(\Omega)^{N}}\left(\left\|\alpha^{-1}\right\|_{B M O\left(\mathbb{R}^{N}\right)}+\left\|\alpha^{-1}\right\|_{L^{1}(\Omega)}^{q}\right)^{\frac{1}{q}}\left\|y_{k}\right\|_{\mathbb{X}_{u, B_{k}}} .
\end{aligned}
$$

Hence, the sequence $\left\{y_{k}\right\}_{k \in \mathbb{N}}$ is bounded in variable space $\mathbb{X}_{u, B_{k}}$,

$$
\begin{aligned}
\left\|y_{k}\right\|_{\mathbb{X}_{u, B_{k}}} & \leq\left(C_{Q, q}\|f\|_{L^{\infty}(\Omega)^{N}}\right)^{\frac{1}{p-1}} \\
& \times\left(\left\|\alpha^{-1}\right\|_{B M O\left(\mathbb{R}^{N}\right)}+\left\|\alpha^{-1}\right\|_{L^{1}(\Omega)}^{q}\right)^{\frac{1}{p}}, \quad \forall k \in \mathbb{N},
\end{aligned}
$$

and, by Lemma 4.3 , we can suppose the existence of an element $y \in \mathbb{X}_{u, B}$ such that (within a subsequence) $y$ is subjected to the estimate (4.20) and

$$
y_{k} \rightarrow y \text { in } L^{p}(\Omega, u d x),
$$

$\nabla y_{k} \rightarrow \nabla y$ in the variable space $L^{p}\left(\Omega, B_{k} d x\right)^{N}$. 
We are now in a position to pass to the limit in (4.24) as $k \rightarrow \infty$. With that in mind we make use of Lemma 4.2. In particular, we utilize the properties (4.6)(4.7). Then, it follows from Definition 2.2 and (4.26)-(4.27) that

$$
\begin{aligned}
\int_{\Omega}\left(f, \nabla \varphi-\nabla y_{k}\right) d x & =\int_{\Omega}\left(B_{k}^{-1} f, B_{k}\left(\nabla \varphi-\nabla y_{k}\right)\right) d x \\
\stackrel{k \rightarrow \infty}{\rightarrow} \int_{\Omega}\left(B^{-1} f, B(\nabla \varphi-\nabla y)\right) d x=\int_{\Omega}(f, \nabla \varphi-\nabla y) d x, & \\
\int_{\Omega}\left|\left(\nabla \varphi, B_{k} \nabla \varphi\right)\right|^{\frac{p-2}{2}} & \left(A_{k} \nabla \varphi, \nabla \varphi-\nabla y_{k}\right) d x \\
& =\int_{\Omega}\left(\left|\left(\nabla \varphi, B_{k} \nabla \varphi\right)\right|^{\frac{p-2}{2}} L_{k}^{-1} T_{k} L_{k} \nabla \varphi, B_{k}\left(\nabla \varphi-\nabla y_{k}\right)\right) d x \\
& \stackrel{k \rightarrow \infty}{\rightarrow} \int_{\Omega}\left(|(\nabla \varphi, B \nabla \varphi)|^{\frac{p-2}{2}} L^{-1} T L \nabla \varphi, B\left(\nabla \varphi-\nabla y_{k}\right)\right) d x \\
& =\int_{\Omega}|(\nabla \varphi, B \nabla \varphi)|^{\frac{p-2}{2}}(A \nabla \varphi, \nabla \varphi-\nabla y) d x .
\end{aligned}
$$

Taking into account that

$$
\int_{\Omega}|\varphi|^{p-2} \varphi\left(\varphi-y_{k}\right) u d x \stackrel{k \rightarrow \infty}{\longrightarrow} \int_{\Omega}|\varphi|^{p-2} \varphi(\varphi-y) u d x
$$

by (4.26) and definition of the weak convergence in $L^{p}(\Omega, u d x)$, we can pass to the limit in (4.24) as $k \rightarrow \infty$ and readily obtain the desired relation (3.9). Thus, $y$ is a weak solution to the boundary value problem (3.1)-(3.3). As for the energy inequality (4.21), it follows from (4.23) and the weak convergence properties (4.26)-(4.27).

Remark 4.2. As follows from approximation procedure that was used in the proof of Theorem 4.1, it always leads to some weak solution of the original boundary value problem. Such solutions are called approximation solutions in [33]. The characteristic feature of such solutions is the fact that they satisfy energy inequality (4.21) and their a priori estimate (4.20) does not depend on the skewsymmetric part $D \in B M O\left(\Omega ; \mathbb{S}_{\text {skew }}^{N}\right)$ of matrix $A \in \mathfrak{M}_{a d}(\Omega)$. Moreover, it is unknown in general whether approximation solutions are the weak solutions to the boundary value problem (3.1)-(3.2) in the sense of distributions and belong to the set $D\left(\mathbb{X}_{u, B}\right)$.

\section{On Density of Smooth Compactly Supported Functions in $W_{0, B}^{1, p}(\Omega)$}

The aim of this section is to find out the sufficient conditions guaranteeing the equality $H_{0, B}^{1, p}(\Omega)=W_{0, B}^{1, p}(\Omega)$. With that in mind, it is enough to check whether, 
for each $A \in \mathfrak{M}_{a d}(\Omega)$ and $p \geq 2$, the set of smooth compactly supported functions $C_{0}^{\infty}(\Omega)$ is dense in $W_{0, B}^{1, p}(\Omega)$.

Let $f \in W_{0, B}^{1, p}(\Omega)$ be an arbitrary function. For any $\delta>0$, we set

$$
\begin{aligned}
\Omega_{\delta} & :=\{x \in \Omega: \operatorname{dist}(x, \partial \Omega)>\delta\} \\
\text { and } \zeta_{\delta}(x) & =\int_{\Omega_{3 \delta / 4}} \omega_{\delta / 4}(|x-y|) d y, \quad \forall x \in \mathbb{R}^{N},
\end{aligned}
$$

where

$$
\omega(x)=\left\{\begin{array}{cc}
C \exp \left(\frac{1}{|x|^{2}-1}\right), & 0 \leq|x|<1, \\
0, & \mid \| \geq 1,
\end{array}\right.
$$

with

$$
C=\left(\int_{B_{1}(0)} \exp \left(\frac{1}{|x|^{2}-1}\right) d x\right)^{-1}
$$

and

$$
\omega_{\delta}(|x|)=\frac{1}{\delta} \omega(|x| / \delta), \quad \forall x \in \mathbb{R}^{N},
$$

so that $\omega_{\delta} \in C_{0}^{\infty}\left(B_{\delta}(0)\right), \int_{\mathbb{R}^{N}} \omega_{\delta}(x) d x=1, \omega_{\delta}(|x|) \geq 0 \forall x \in \mathbb{R}^{N}$.

Then, the following properties of $\zeta_{\delta}$ are well-known [24, Theorem 1.4.2]:

(i) $0 \leq \zeta_{\delta}(x) \leq 1$ for all $x \in \mathbb{R}^{N}$;

(ii) $\zeta_{\delta}(x)=1$ for all $x \in \Omega_{\delta}$;

(iii) $\zeta_{\delta}(x)=0$ outside of $\Omega_{\delta / 2}$; (iv) $\left|\frac{\partial \zeta_{\delta}(x)}{\partial x_{i}}\right| \leq \frac{C}{\delta} \forall x \in \mathbb{R}^{N}, i=1, \ldots, N$, where $C$ is a positive constant
independent of $\delta$.

Setting $f^{\delta}(x):=f(x) \zeta_{\delta}(x)$, we see that $f^{\delta}=0$ outside of $\Omega_{\delta / 2}$. Before proceeding further, we make use of the following auxiliary result.

Lemma 5.1. Assume that, in addition to (2.2)-(2.3), the functions $\alpha$ and $\beta$ satisfy the condition

$$
\alpha^{-1}, \beta \in L^{\infty}\left(\Omega \backslash \Omega_{\delta}\right), \quad \text { where } \Omega_{\delta}:=\{x \in \Omega: \operatorname{dist}(x, \partial \Omega)>\delta\}
$$

for some $\delta>0$ small enough. Then for given $A \in \mathfrak{M}_{a d}(\Omega)$ and $f \in W_{0, B}^{1, p}(\Omega)$, we have

$$
f^{\delta} \in W_{0, B}^{1, p}(\Omega) \quad \text { and } \quad\left\|f-f^{\delta}\right\|_{W_{0, B}^{1, p}(\Omega)}^{p}=o(1) \quad \text { as } \delta \rightarrow 0 .
$$


Proof. Indeed, the inclusion $f^{\delta} \in W_{0, B}^{1, p}(\Omega)$ is a direct consequence of the property $f^{\delta}=0$ outside of $\Omega_{\delta / 2}$ and the following estimate

$$
\begin{aligned}
\left\|f^{\delta}\right\|_{W_{0, B}^{1, p}(\Omega)}^{p} & =\int_{\Omega}\left(\left|f^{\delta}\right|^{p}+\left|\left(\nabla f^{\delta}, B \nabla f^{\delta}\right)\right|^{\frac{p}{2}}\right) d x \\
& =\int_{\Omega}\left(\left|f \zeta_{\delta}\right|^{p}+\left|L\left(\zeta_{\delta} \nabla f+f \nabla \zeta_{\delta}\right)\right|^{p}\right) d x \\
& \leq \int_{\Omega}\left(|f|^{p}+p|L \nabla f|^{p}+p|f|^{p} \beta^{p}\left|\nabla \zeta_{\delta}\right|^{p}\right) d x \\
& \leq(1+p)\|f\|_{W_{0, B}^{1, p}(\Omega)}^{p}+p\|\beta\|_{L^{\infty}\left(\Omega \backslash \Omega_{\delta}\right)}^{p}\left(\sqrt{\frac{C^{2} N}{\delta^{2}}}\right)^{p} \int_{\Omega \backslash \Omega_{\delta}}|f|^{p} d x \\
& \leq C(\delta)\|f\|_{W_{0, B}^{1, p}(\Omega)}^{p}
\end{aligned}
$$

which is valid for $\delta$ small enough (see (5.1)).

As for the asymptotic behaviour of the difference $f-f \zeta_{\delta}=f\left(1-\zeta_{\delta}\right)$, we provide this analysis utilizing the following chain of estimates

$$
\begin{aligned}
\left\|f-f \zeta_{\delta}\right\|_{W_{0, B}^{1, p}(\Omega)}^{p} & =\int_{\Omega}\left|f\left(1-\zeta_{\delta}\right)\right|^{p} d x+\int_{\Omega}\left|\left(1-\zeta_{\delta}\right) L(\nabla f)-f L\left(\nabla \zeta_{\delta}\right)\right|^{p} d x \\
\leq & \int_{\Omega \backslash \Omega_{\delta}}|f|^{p} d x+p \int_{\Omega \backslash \Omega_{\delta}}|(\nabla f, B \nabla f)|^{\frac{p}{2}} d x \\
& \quad+p \int_{\Omega \backslash \Omega_{\delta}}|f|^{p} \beta^{p}\left|\nabla \zeta_{\delta}\right|^{p} d x \\
\leq & (1+p)\|f\|_{W_{0, B}^{1, p}\left(\Omega \backslash \Omega_{\delta}\right)}^{p} \\
& +p\|\beta\|_{L^{\infty}\left(\Omega \backslash \Omega_{\delta}\right)}^{p}\left(\frac{C \sqrt{N}}{\delta}\right)^{p} \int_{\Omega \backslash \Omega_{\delta}}|f|^{p} d x
\end{aligned}
$$

In order to estimate the last term in (5.3), we make use of the Maz'ya inequality (2.23). This gets

$$
\begin{aligned}
& \left(\int_{\Omega \backslash \Omega_{\delta}}|f|^{p} d x\right)^{\frac{1}{p}} \leq \sup _{\Omega^{\prime} \subset \Omega \backslash \Omega_{\delta}} \frac{\mathcal{L}^{N}\left(\Omega^{\prime}\right)^{\frac{1}{p}}}{\mathcal{H}^{N-1}\left(\partial \Omega^{\prime}\right)} \int_{\Omega \backslash \Omega_{\delta}}|\nabla f| d x \\
& \quad \leq \sup _{\Omega^{\prime} \subset \Omega \backslash \Omega_{\delta}} \frac{\mathcal{L}^{N}\left(\Omega^{\prime}\right)^{\frac{1}{p}}}{\mathcal{H}^{N-1}\left(\partial \Omega^{\prime}\right)} \int_{\Omega \backslash \Omega_{\delta}}|L \nabla f| \alpha^{-1} d x \\
& \quad \leq \sup _{\Omega^{\prime} \subset \Omega \backslash \Omega_{\delta}} \frac{\mathcal{L}^{N}\left(\Omega^{\prime}\right)^{\frac{1}{p}}}{\mathcal{H}^{N-1}\left(\partial \Omega^{\prime}\right)}\left\|\alpha^{-1}\right\|_{L^{\infty}\left(\Omega \backslash \Omega_{\delta}\right)} \mathcal{L}^{N}\left(\Omega^{\prime}\right)^{\frac{1}{q}}\left(\int_{\Omega \backslash \Omega_{\delta}}|L \nabla f|^{p} d x\right)^{\frac{1}{p}} \\
& \quad \leq\left\|\alpha^{-1}\right\|_{L^{\infty}\left(\Omega \backslash \Omega_{\delta}\right)} \sup _{\Omega^{\prime} \subset \Omega \backslash \Omega_{\delta}} \frac{\mathcal{L}^{N}\left(\Omega^{\prime}\right)}{\mathcal{H}^{N-1}\left(\partial \Omega^{\prime}\right)}\|f\|_{W_{0, B}^{1, p}\left(\Omega \backslash \Omega_{\delta}\right)}
\end{aligned}
$$


Since $\mathcal{L}^{N}\left(\Omega^{\prime}\right) \leq C^{*} \delta \mathcal{H}^{N-1}\left(\partial \Omega^{\prime}\right)$ for $\delta$ small enough and with $C^{*}$ independent of $\delta$, it follows from (5.4) that

$$
\int_{\Omega \backslash \Omega_{\delta}}|f|^{p} d x \leq \operatorname{const} \delta^{p}\|f\|_{W_{0, B}^{1, p}\left(\Omega \backslash \Omega_{\delta}\right)}^{p} .
$$

Thus, from (5.3) we finally deduce

$$
\left\|f-f \zeta_{\delta}\right\|_{W_{0, B}^{1, p}(\Omega)}^{p} \leq \widehat{C}\|f\|_{W_{0, B}^{1, p}\left(\Omega \backslash \Omega_{\delta}\right)}^{p}=o(1) \text { as } \delta \rightarrow 0 .
$$

Taking this result into account and following the standard rule, we define the smoothing of $f^{\delta}$ :

$$
\left(f \zeta_{\delta}\right)_{\varepsilon}(x):=\int_{\mathbb{R}^{N}} \omega_{\varepsilon}(x-y) f(y) \zeta_{\delta}(y) d y=\left(\omega_{\varepsilon} * f^{\delta}\right)(x), \quad \forall x \in \mathbb{R}^{N} .
$$

Then $\left(f \zeta_{\delta}\right)_{\varepsilon}(x)=0$ has a compact support in $\Omega$ provided $\varepsilon<\delta / 2$. Since $\left(f \zeta_{\delta}\right)_{\varepsilon} \in$ $C_{0}^{\infty}(\Omega)$ and $W_{0, B}^{1, p}(\Omega) \subset W^{1, p_{s}}(\Omega)$ with continuous embedding for all $p_{s}<p$ (see estimates $(2.14)-(2.15))$, it follows from the classical theory of Sobolev spaces that $\left(f \zeta_{\delta}\right)_{\varepsilon} \rightarrow f \zeta_{\delta}$ in $W^{1, p_{s}}(\Omega)$ as $\varepsilon \rightarrow 0$ and, therefore, up to a subsequence, we can suppose that $\left(f \zeta_{\delta}\right)_{\varepsilon} \rightarrow f \zeta_{\delta}$ almost everywhere in $\Omega$. Let us show that $\left(f \zeta_{\delta}\right)_{\varepsilon} \rightarrow f$ in $W_{0, B}^{1, p}(\Omega)$. Indeed, we can deduce from (5.6) that

$$
\left|\nabla\left(f^{\delta}\right)_{\varepsilon}(x)\right| \leq C_{1} M\left(\nabla f^{\delta}\right)(x), \quad \forall \varepsilon>0,
$$

where $M(f)(x)=\sup _{Q} \frac{1}{|Q|} \int_{Q}|f(y)| d y$ is the Hardy-Littlewood maximal function. It is also known that [12, p.174]

$$
\alpha, 1 / \alpha \in \bigcap_{r>1} A_{r} \quad \Leftrightarrow \quad \ln \alpha \in \operatorname{closure}_{B M O} L^{\infty}\left(\mathbb{R}^{N}\right) .
$$

Since $\ln \alpha \in$ closure $_{B M O} L^{\infty}\left(\mathbb{R}^{N}\right)$ is equivalent to $\ln \alpha^{p} \in \operatorname{closure}_{B M O} L^{\infty}\left(\mathbb{R}^{N}\right)$, it follows from (5.8) and (2.2)-(2.3) that $\alpha^{p}, \beta^{p} \in A_{p}$. Then, by the celebrated Mackengoupt theorem [22], we have

$$
\begin{aligned}
& \alpha^{p} \in A_{p} \quad \Leftrightarrow \quad \int_{\mathbb{R}^{N}}\left|M\left(\nabla f^{\delta}\right)\right|^{p} \alpha^{p} d x \leq C(\alpha, p) \int_{\mathbb{R}^{N}}\left|\nabla f^{\delta}\right|^{p} \alpha^{p} d x, \\
& \beta^{p} \in A_{p} \quad \Leftrightarrow \quad \int_{\mathbb{R}^{N}}\left|M\left(\nabla f^{\delta}\right)\right|^{p} \beta^{p} d x \leq C(\beta, p) \int_{\mathbb{R}^{N}}\left|\nabla f^{\delta}\right|^{p} \beta^{p} d x .
\end{aligned}
$$

Since the norms $|\xi|$ and $\sqrt{(\xi, B \xi)}$ are equivalent in $\mathbb{R}^{N}$, it follows that

$$
\begin{aligned}
\beta^{p}, \alpha^{p} \in A_{p} \Leftrightarrow \int_{\mathbb{R}^{N}}\left|\left(M\left(\nabla f^{\delta}\right), B M\left(\nabla f^{\delta}\right)\right)\right|^{\frac{p}{2}} d x & \\
& \leq C_{2} \int_{\mathbb{R}^{N}}\left|\left(\nabla f^{\delta}, B \nabla f^{\delta}\right)\right|^{\frac{p}{2}} d x
\end{aligned}
$$


for some positive constant $C_{2}$ depending on $\alpha, \beta$, and $p$. Using the fact that each of the matrices $A \in \mathfrak{M}_{a d}(\Omega)$ is assumed to be zero-extended outside of $\Omega$, we deduce from (5.7) and (5.9)

$$
\begin{aligned}
\int_{\Omega} \mid\left(\nabla\left(f^{\delta}\right)_{\varepsilon},\right. & \left.B \nabla\left(f^{\delta}\right)_{\varepsilon}\right)\left.\right|^{\frac{p}{2}} d x=\int_{\mathbb{R}^{N}}\left|\left(\nabla\left(f^{\delta}\right)_{\varepsilon}, B \nabla\left(f^{\delta}\right)_{\varepsilon}\right)\right|^{\frac{p}{2}} d x \\
\leq & C \int_{\mathbb{R}^{N}}\left|\left(\nabla f^{\delta}, B\left(\nabla f^{\delta}\right)\right)\right|^{\frac{p}{2}} d x \\
& =C \int_{\Omega}\left|\left(\nabla f^{\delta}, B\left(\nabla f^{\delta}\right)\right)\right|^{\frac{p}{2}} d x \leq C\left\|f^{\delta}\right\|_{W_{0, B}^{1, p}(\Omega)}^{p}<+\infty .
\end{aligned}
$$

Following the similar reasoning, it can be shown that

$$
\int_{\Omega}\left|\left(f^{\delta}\right)_{\varepsilon}\right|^{p} d x \leq C \int_{\Omega}\left|f^{\delta}\right|^{p} d x \leq C\left\|f^{\delta}\right\|_{W_{0, B}^{1, p}(\Omega)}^{p}<+\infty .
$$

Hence, the sequence $\left\{\left(f^{\delta}\right)_{\varepsilon}\right\}_{\varepsilon>0}$ is bounded in $\|\cdot\|_{W_{0, B}^{1, p}(\Omega)}$-norm. Therefore, in view of the pointwise convergence: $\left(f \zeta_{\delta}\right)_{\varepsilon} \rightarrow f \zeta_{\delta}$ almost everywhere in $\Omega$, we can deduce the weak convergence $\left(f \zeta_{\delta}\right)_{\varepsilon} \rightarrow f \zeta_{\delta}$ in $W_{0, B}^{1, p}(\Omega)$. Then by Mazur's theorem, the element $f^{\delta}:=f \zeta_{\delta}$ can be attained in the strong topology of $W_{0, B}^{1, p}(\Omega)$ by the convex combinations of $\left\{\left(f^{\delta}\right)_{\varepsilon}\right\}_{\varepsilon>0}$. It means that for any given $\eta>0$ it can be found a convex combination $f_{*}^{\delta} \in C_{0}^{\infty}(\Omega)$ of a finite number of elements of the sequence $\left\{\left(f^{\delta}\right)_{\varepsilon}\right\}_{\varepsilon>0}$ such that

$$
\left\|f_{*}^{\delta}-f^{\delta}\right\|_{W_{0, B}^{1, p}(\Omega)}<\frac{\eta}{2}
$$

Besides, the property (5.2) implies that

$$
\left\|f-f^{\delta}\right\|_{W_{0, B}^{1, p}(\Omega)}<\frac{\eta}{2} \quad \text { for } \delta \text { small enough. }
$$

Hence, for a given function $f \in W_{0, B}^{1, p}(\Omega)$ and arbitrary positive $\eta$, we have

$$
\left\|f-f_{*}^{\delta}\right\|_{W_{0, B}^{1, p}(\Omega)}<\eta .
$$

Thus, we can formulate the obtained result as follows:

Theorem 5.1. Assume the set of admissible matrices $\mathfrak{M}_{a d}(\Omega)$ is such that in addition to its definition in the form (2.5), the condition (5.1) holds true for some positive small enough parameter $\delta$. Then the set of smooth compactly supported functions $C_{0}^{\infty}(\Omega)$ is dense in $W_{0, B}^{1, p}(\Omega)$ or, what is equivalent, we have the equality $H_{0, B}^{1, p}(\Omega)=W_{0, B}^{1, p}(\Omega)$. 


\section{References}

1. D.J. Bergman, D. Stroud, Physical properties of macroscopically inhomogeneous media, North-HollaSolid State Physics, 46 (1992), 147-269.

2. M. BRiAne, J. CASADO-Diaz, Uniform convergence of sequences of solutions of two-dimensional linear elliptic equations with unbounded coefficients, J. of Diff. Equa., 245 (2008), 2038-2054.

3. D. Bucur, G. Buttazzo, Variational Methodth in Shape Optimization Problems, Birkhäuser, Boston, 2005.

4. P. Caldiroli, R. Musina, On a variational degenerate elliptic problem, Nonlinear Diff. Equa. Appl., 7 (2000), 187-199.

5. V. Chiado Piat, F. Serra Cassano, Some remarks about the density of smooth functions in weighted Sobolev spaces, J. Convex Analysis, No. 2, 1 (1994), 135-142.

6. M. Chicco, M. Venturino, Dirichlet problem for a divergence form elliptic equation with unbounded coefficients in an unbounded domain, Annali di Matematica Pura ed Applicata, 178 (2000), 325-338.

7. C. D'Apice, U. De Maio, P.I. Kogut, R. Manzo, Solvability of an optimal control problem in coefficients for ill-posed elliptic boundary value problems, Electronic Journal of Differential Equations, 2014 (166) (2014), 1-23.

8. C. D'Apice, U. De MAIO, O. P. Kogut, Optimal control problems in coefficients for degenerate equations of monotone type: shape stability and attainability problems, SIAM J. Control Optim., 50 (3) (2012) 1174-1199.

9. C. D'Apice, U. De Maio, O. P. Kogut, On shape stability of Dirichlet optimal control problems in coefficients for nonlinear elliptic equations, Adv. Differential Equations, 15 (7-8) (2010) 689-720.

10. P. Drabek, A. Kufner, F. Nicolosi, Non-Linear Elliptic Equations, Singular and Degenerate Cases, Walter de Cruyter, Berlin, 1997.

11. T. Durante, O. P. Kupenko, R. Manzo, On attainability of optimal controls in coefficients for system of Hammerstein type with anisotropic p-Laplacian, Ricerche di Matematica, 66 (2) (2017) 259-292.

12. J. Garcia-Cuerva, J.L. Rubio De Francia, Weighted Norm Inequalities and Related Topics, North Holland Math. Studies, Vol.116, Amsterdam, North-Holland, 1985.

13. F. John, L. Nirenberg, On functions of bounded mean oscillation, Comm. Pure Appl. Math., 14(1961), 415-426.

14. T. Horsin, P.I. Kogut, Optimal $L^{2}$-control problem in coefficients for a linear elliptic equation. I. Existence result, Mathematical Control and Related Fields, 5 (1) (2015), 73-96.

15. T. Horsin, P.I. Kogut, On unbounded optimal controls in coefficients for ill-posed elliptic Dirichlet boundary value problems, Asymptotic Analysis, 98 (1-2) (2016), 155-188.

16. P.I. KogUT, On approximation of an optimal boundary control problem for linear elliptic equation with unbounded coefficients, Discrete and Continuous Dynamical Systems - Series A, 34 (5) (2014), 2105-2133.

17. P.I. Kogut, G. Leugering, Optimal Control Problems for Partial Differential Equations on Reticulated Domains. Approximation and Asymptotic Analysis, Series: Systems and Control, Birkhäuser Verlag, Boston, 2011.

18. P.I. Kogut, G. Leugering, Matrix-valued $L^{1}$-optimal control in the coefficients of linear elliptic problems, Journal for Analysis and its Applications (ZAA), 32 (4) (2013), 433-456. 
19. O.P. Kupenko, R. Manzo, Approximation of an optimal control problem in coefficient for variational inequality with anisotropic p-Laplacian, Nonlinear Differential Equations and Applications (NoDEA), 23 (3) (2016) 1-18.

20. O.P. Kupenko, R. Manzo, On optimal controls in coefficients for ill-posed nonlinear elliptic Dirichlet bounday value problems, Discrete and Continuous Dynamical Systems, Series B, 23 (4) (2018), 1363-1393.

21. O. Levy, R. V. KoHn, Duality relations for non-ohmic composites, with applications to behavior near percolation, J. Statist. Phys., 90 (1998), 159-189.

22. B. Mackengoupt, Weighted norm inequalities for Hardy maximal function, Trans. Amer. Math. Soc., 165 (1972), 207-226.

23. V.G. MAZ'YA, On cetrtain integral inequalities for functions nof many variables, J. Soviet Math., 1 (1973), 205-234.

24. V.P. Mihaylov, A.K. Gustchin, Supplemental Chapters to the Course 'Equations of Mathematical Physics', Lecture Cources of the Steklov Mathematical Institute of Russian Academy of Sciences, 7, Moskow, MIAN, 2007 (in Russian).

25. S.E. Pastuknova, Degenerate equations of monotone type: Lavrent'eff phenomenon and attainability problems, Sbornik: Mathematics, 198 (10) (2007), 1465-1494.

26. F. Punzo, A. TESEI, Uniqueness of solutions to degenerate elliptic problems with unbounded coefficients, Ann. I.H. Poincaré, 26 (2009), 2001-2024.

27. T. RADICE, Regularity result for nondivergence elliptic equations with unbounded coefficients, Diff. Integral Equa., 23 (9-10) (2010), 989-1000.

28. T. RADICE, G. ZECCA, Existence and uniqueness for nonlinear elliptic equations with unbounded coefficients, Ricerche Mat., 63 (2014), 355-267.

29. T. RoubičEk, Nonlinear Partial Differential Equations with Applications, Birkhäuser, Basel, 2013.

30. E. SaAcson, H. B. Keller, Analysis of Numerical Methods, Wiley, New York, 1966.

31. M.V. SAFonov, Non-divergence elliptic equations of second order with unbounded drift, Nonlinear Partial Diff. Equa. and Related Topics, 229 (2) (2010), 211-232.

32. G. ZECCA, An optimal control problem for nonlinear elliptic equations with unbounded coefficients, to appear on Discrete and Continous Dynamical Systems, Series B, (2019).

33. V.V. Zhikov, Remarks on the uniqueness of a solution of the Dirichlet problem for second-order elliptic equations with lower-order terms, Functional Analysis and Its Applications, 38 (3) (2004), 173-183. 